\title{
Automatic classification of retinal blood vessels based on multilevel thresholding and graph propagation
}

\author{
Beatriz Remeseiro • Ana Maria \\ Mendonça • Aurélio Campilho
}

Received: date / Accepted: date

\begin{abstract}
Several systemic diseases affect the retinal blood vessels, thus their assessment allows an accurate clinical diagnosis. This assessment entails the estimation of the arteriolar-to-venular ratio (AVR), a predictive biomarker of cerebral atrophy and cardiovascular events in adults. In this context, different automatic and semi-automatic image-based approaches for artery/vein (A/V) classification and AVR estimation have been proposed in the literature, to the point of having become a hot research topic in the last decades. Most of these approaches use a wide variety of image properties, often redundant and/or irrelevant, requiring a training process that limit their generalization ability when applied to other datasets. This paper presents a new automatic method for $\mathrm{A} / \mathrm{V}$ classification that just uses the local contrast between blood vessels and their surrounding background, computes a graph that represents the vascular structure, and applies a multilevel thresholding to obtain a preliminary classification. Next, a novel graph propagation approach was developed to obtain the final A/V classification and to compute the AVR. Our approach has been tested on two public datasets (INSPIRE and DRIVE), obtaining high classification accuracy rates, specially in the main vessels, and AVR ratios very similar to those provided by human experts. Therefore, our fully automatic method provides reliable results without any training step, which makes it suitable for use with different retinal image datasets and as part of any clinical routine.
\end{abstract}

Beatriz Remeseiro · Ana Maria Mendonça · Aurélio Campilho

INESC TEC - INESC Technology and Science. Campus da FEUP, Rua Dr. Roberto Frias, 4200-465 Porto, Portugal.

Present address (Beatriz Remeseiro): Department of Computer Science, Universidad de Oviedo. Campus de Gijón s/n, 33203 Gijón, Spain.

E-mail: bremeseiro@uniovi.es

Ana Maria Mendonça · Aurélio Campilho

Faculdade de Engenharia, Universidade do Porto. Campus da FEUP, Rua Dr. Roberto Frias, 4200-465 Porto, Portugal.

E-mail: amendon@fe.up.pt, campilho@fe.up.pt 
Keywords retinal images · artery/vein classification · arteriolar-to-venular ratio $\cdot$ multilevel thresholding $\cdot$ graph propagation

\section{Introduction}

Human blood circulation can be observed in-vivo in the eye, allowing to diagnose several systemic diseases that affect the retinal vessels in such a way that they become thicker or narrower $[1,2]$. Some of these diseases include diabetes, with a $8.5 \%$ of global prevalence among adults according to the $\mathrm{WHO}^{1}$; raised blood pressure, which is estimated to cause about the $12.8 \%$ of the total of all deaths worldwide as reported by WHO; and different vascular disorders. More specifically, diabetic retinopathy frequently causes vessel diameter alterations [3], whilst dilatation and elongation of main arteries and veins are often associated with hypertension and other cardiovascular pathologies [4].

In this context, the arteriolar-to-venular ratio (AVR) plays an important role since it has been shown to be associated with several risk factors such as cardiovascular or inflammatory biomarkers [5], and correlated with different diseases including stroke or atherosclerosis [6]. The AVR represents the relationship between the calibers of both arteries and vein, measured within a standard ring area around the optical disc. Consequently, the automatic calculation of the AVR requires an accurate classification of blood vessels into arteries and veins, also known as A/V classification, which has become a leading topic in retinal image analysis over the last years [7].

Arteries are brighter and thinner than veins, and the central reflex at the inner part is more obvious in arteries. This difference between arteries and veins has been used in many A/V classification approaches to assign A/V labels to vessel pixels. In this sense, Montoro et al. [8] analyzed the appearance of the retinal tree in different color spaces, including RGB and HSV, to extract relevant features such as the hue mean, the variance of the red contrast and the mean of the saturation contrast. Irshad et al. [9] presented an automatic method for $\mathrm{A} / \mathrm{V}$ classification based on intensity and gradient features. Using only four color features, Relan et al. [10] proposed a squared-loss mutual information clustering to perform the retinal vessel classification. An early diagnostic tool for various diseases was presented by $\mathrm{Xu}$ et al. [11], using not only color features extracted from the CIE xyY color space, but also texture features based on both first- and seconder-order statistics. With the final target of detecting hypertensive retinopathy, Akbar et al. [12] proposed the used of a hybrid set of features that includes both color and statistical based textural features for A/V classification. Huang et al. [13] proposed a wide set of features and then applied a feature selection method based on genetic-search. In this manner, they obtained a subset of features that fed a linear discriminant analysis for the final $\mathrm{A} / \mathrm{V}$ classification.

In addition to image properties, the structural information of both arteries and veins in the retinal tree has also been considered for $\mathrm{A} / \mathrm{V}$ classification. For

1 World Health Organization: http://www.who.int/en/ 
example, Mirsharif et al. [14] presented a structural method for blood vessel classification in which intersection and bifurcation points were processed, after computing features such as vessel widths or pixel intensities in different color spaces (RGB, HSL, and LAB). In the field of structural information, graphbased approaches are also commonly found in the literature. Graph search was used in [15] as part of an automatic method for retinal vessel identification, in which features such as orientation, width and intensity of each vessel segment were used to find the optimal graph. A different approach for A/V classification was presented in [16], whose authors used the graph extracted from the retinal vasculature combined with a set of intensity features. Estrada et al. [17] proposed a graph-theoretic framework using the vessel tree topology along with domain-specific features. On the other hand, a more global framework was proposed by $\mathrm{Hu}$ et al. [18], who generated a vessel network composed of vessel segments and their potential connectivity, and made use of a graph-based meta-heuristic algorithm for the final A/V classification. In this context, although applied to ultra-wide-field-of-view retinal images, Pellegrini et al. [19] proposed a graph cut approach for A/V classification by using hand-crafted features, which include local vessel intensity and vascular morphology. More recently, Zhao et al. [20] adapted the concept of dominant set clustering to estimate the vascular network of the retinal blood vessels, and used intensity and morphology features to finally classify its pixels.

Deep learning techniques started to be applied to the A/V classification problem. Meyer et al. [21] proposed a novel approach based on convolutional neural networks $(\mathrm{CNN})$ to classify the pixels belonging to the retinal vasculature tree into arteries and veins. Some popular datasets include vessels pixels manually labeled not only as artery or vein, but also as uncertain. This uncertainty is due to the difficulty found by the specialists to label some vessel pixels in retinal images, mainly because of the limitations of acquisition devices. In this context, Galdran et al. [22] proposed a CNN trained to classify the pixels into one of four classes (background, artery, vein, and uncertain), thus providing an automatic segmentation of the vasculature tree.

Regarding the computation of the AVR, Niemeijer et al. [23] presented an automatic method for AVR estimation where arteries and veins were classified by means of an iterative algorithm that used centerline pixel features and crossing/bifurcation points of the vascular tree. In [24], an AVR monitoring system was presented, which analyzed different images from the same patient using a registration approach to measure the vessel widths at the same points in all the images. Three automatic approaches for the estimation of the AVR were compared in [25], defined as different methodologies for optic disc and vessel segmentation, vessel caliber estimation, and A/V classification.

Improving the illumination properties of retinal images for a more accurate $\mathrm{A} / \mathrm{V}$ classification was also the focus of attention in several recent works. Mustafa et al. [26] proposed a new approach, based on low pass and Gaussian filters, to correct the illumination. Varnousfaderani et al. [27] presented a method for non-uniform illumination removal by normalizing the luminance using the LUV color space, and for contrast enhancement by histogram shift- 
ing. Huang et al. [28] presented a new normalization technique that allows to compute four new features related to the lightness reflection of vessels, adequate for $\mathrm{A} / \mathrm{V}$ classification.

\subsection{Rationale of the approach}

Most of the previous approaches use a wide variety of features, ranging from the 3 features suggested by Estrada et al. [17] to the 100 proposed by Huang et al. [13]. These sets of features are often redundant and/or irrelevant, as evidenced by some research works that applied feature selection techniques to select the optimal subset of features $[13,14,16]$. Additionally, Dashtbozorg [29] analyzed the individual impact of each of the 30 features considered, demonstrating that the two most relevant features were extracted from the red component of the RGB color space. In particular, they are two basic features: the red intensity of the centerline pixels, and the standard deviation of the red intensity in the vessels. With respect to the graph-based approaches that represent the vascular tree, they commonly use an initial classification based on different images features, subsequently modified taking into account the graph structure. During this process, a correct classification achieved with the image features can be wrongly modified later due to possible errors made during graph computation. Regarding the technique followed to assign the A/V classes to the vessel pixels, there are supervised and unsupervised approaches. In this sense, it is worth noting that supervised techniques require a training process on a given dataset, thus limiting their generalization ability and providing worse results when applied to other datasets.

In clinical practice, specialists focused not only on the higher intensity of arteries against veins in absolute terms, but mainly on their relative contrast with respect to the surrounding background. For this reason, we propose to use the local contrast as a simple, yet powerful image feature for A/V classification. More specifically, we present a new method, based on multilevel thresholding and graph propagation, to automatically classify blood vessels into arteries and veins, allowing the final computation of an accurate AVR useful for both medical and research purposes. Notice that the graph propagation procedure combines the labels obtained by applying multilevel thresholding to the local contrast feature with the graph structure. In this manner, we can make the most of both steps and thus reduce the cases in which errors in only one of the two steps imply an increase in terms of misclassification rate. Note also that it is an unsupervised method that can be easily applied to any retinal dataset, without requiring a previous training phase. The main contributions of our approach are three-fold: (1) the use of the local contrast as a single feature for $\mathrm{A} / \mathrm{V}$ classification that imitates the perception of specialists, (2) the multilevel image thresholding based on local contrast for a robust initial classification, and (3) a graph propagation procedure that takes full advantage of the multilevel thresholding and the vascular network to provide a final $\mathrm{A} / \mathrm{V}$ classification. 
The remainder of this manuscript is structured as follows: Section 2 presents the approach proposed in this research, Section 3 shows the experimental results obtained using two retinal image datasets and, finally, Section 4 includes the conclusions and future lines of research.

\section{Methods}

The automatic classification of retinal blood vessels requires the detection of several landmarks, allowing the $\mathrm{A} / \mathrm{V}$ classification and the final computation of the AVR. Figure 1 illustrates the whole procedure, where the main contributions are highlighted with a blue background. In the following sections, we make special emphasis on these four main steps, whilst the rest of them are briefly explained and properly referenced.

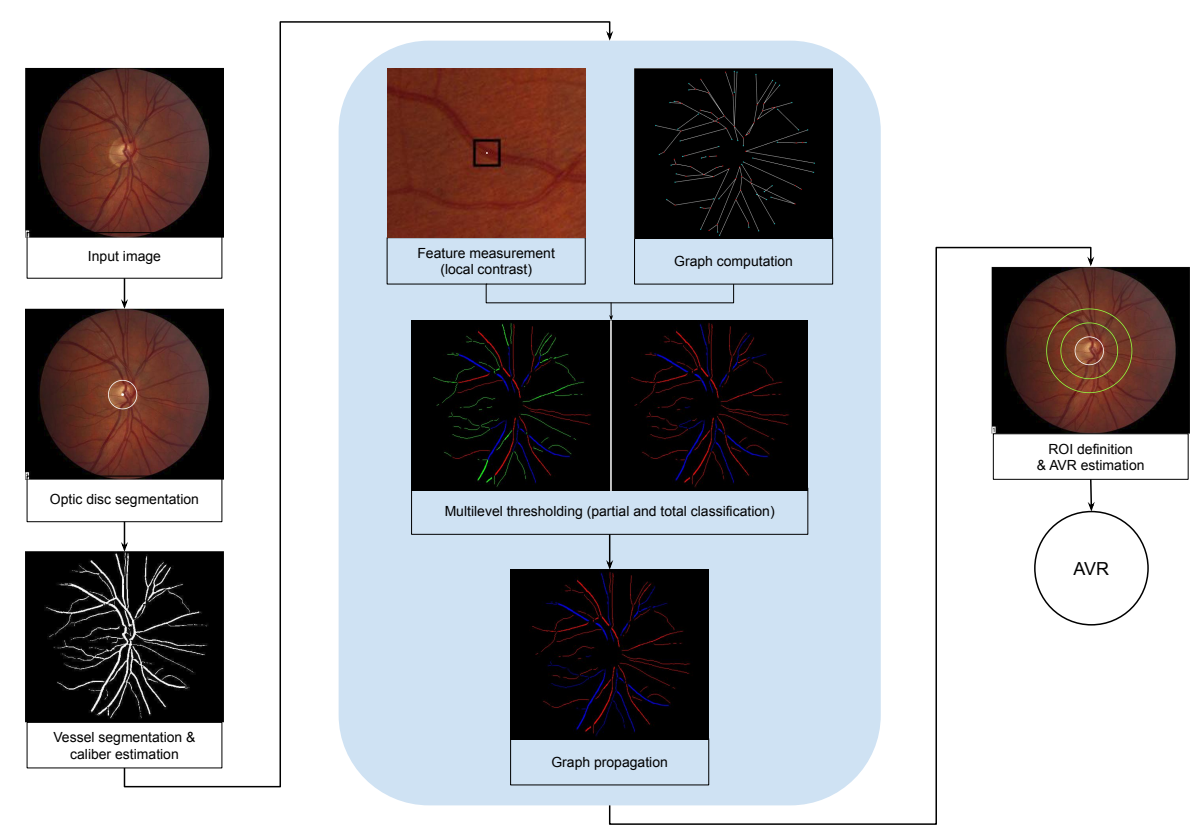

Fig. 1 Workflow of the proposed method for A/V classification and AVR estimation, with the novel steps in blue. Input image from INSPIRE [30].

Broadly speaking, a retinal image is first segmented to obtain the optic disc and the blood vessels. Next, the feature measurement step is carried out to calculate the local contrast of vessel pixels and, in parallel, the vasculature graph is computed. Using both of them, a multilevel thresholding is applied to obtain two A/V classified images: a robust initial classification that includes the uncertain label, and a total classification used for achieving a full coverage rate. Next, the artery/vein labels of the initial classification are propagated to 
those segments classified as uncertain, using the graph structure. Finally, the region of interest is defined and the AVR is estimated.

\subsection{Optic disc segmentation}

The optic disc (OD), one of the main structures visible in a retinal image, is the entry point for the major retinal blood vessels. Its segmentation is an important step in the automatic calculation of the AVR index, as the measuring region is centered in the OD and depends on the OD diameter, as mentioned before.

OD segmentation is carried out following the method proposed in [31], a robust algorithm based on multi-resolution sliding band filters.

\subsection{Vessel segmentation and caliber estimation}

Since the AVR index is calculated from the vessel calibers, another important step is to segment the retinal blood vessels and estimate their calibers.

For the vessel segmentation, the method originally presented in [32] was considered, including the further improvements proposed in [33], which added an increased adaptation to image size and field-of-view, and a decreased sensitivity to parameter settings.

To estimate the vessel calibers, we used the Euclidean distance from the central pixel of the segmented vessels and its closest background pixel.

\subsection{Feature measurement}

On color retinal images, arteries are often brighter than veins since their blood contains, respectively, oxygenated and deoxygenated hemoglobin. Based on this fact, it would seem reasonable to directly use intensity features measured on different channels or color spaces. However, the background of retinal images is not homogeneous and it affects the visual appearance of vessel pixels. For this reason, we propose a novel approach that uses a simple and single feature for artery-vein discrimination: the local contrast. More specifically, this feature represents the local variation measured as the difference between vessel pixel intensities and the background tissue intensities that surrounds them in a local neighborhood.

In order to calculate the local contrast, we considered the red channel of the RGB color space, since it shows the best discrimination power between arteries and veins [29]. Taking into account that the illumination of retinal images is non uniform due to the acquisition procedure, we applied the normalization method proposed by Foracchia et al. [34] to the red channel of the input image. For each vessel pixel $(i, j)$, the local contrast $C$ is calculated as:

$$
C[i, j]=|I[i, j]-B[i, j]|,
$$


where $I$ is the normalized red channel of the original image [29], and $B$ is the average of the background pixels in a neighborhood determined by a $(2 k+1)$ square window centered at $(i, j)$ calculated as:

$$
B[i, j]=\frac{1}{N_{i, j}} \sum_{u=-k}^{k} \sum_{v=-k}^{k} I[i+u, j+v] M[i+u, j+v],
$$

where $N_{i, j}$ is the number of background pixels in the neighborhood:

$$
N_{i, j}=\sum_{u=-k}^{k} \sum_{v=-k}^{k} M[i+u, j+v]
$$

and $M$ is the mask corresponding to the vessel segmented images:

$$
M\left[i^{\prime}, j^{\prime}\right]= \begin{cases}0 & \text { if }\left(i^{\prime}, j^{\prime}\right) \text { is a vessel pixel } \\ 1 & \text { if }\left(i^{\prime}, j^{\prime}\right) \text { is a background pixel. }\end{cases}
$$

\subsection{Graph computation}

The graph extracted from the segmented retinal vasculature proved to be adequate for $\mathrm{A} / \mathrm{V}$ classification [16,17]. This section summarizes the algorithm for graph computation proposed by Dashtbozorg et al. [16], which was improved in this research as further explained.

The original procedure consists of the following main steps [16]:

1. Graph extraction: The vessel centerlines are firstly obtained from the segmented image to finally generate a vascular network composed of nodes and links. Nodes represent the intersection points in the vascular tree, whilst links represent vessel segments between intersection points.

2. Graph modification: Due to the segmentation and centerline extraction processes, the vascular network initially generated may include some misrepresentations. For this reason, the graph is modified to avoid the following common errors: node splitting, missing link, and false link. Next, all vessels around the optic disc are removed since, in this area, the vessels are not relevant for the AVR estimation and the graph may not be reliable.

3. Node type decision: Once the final graph is obtained, the nodes are classified into different categories: connecting point, meeting point, bifurcation point, and crossing point. For this purpose, we use the degree of a node (i.e., the number of adjacent nodes) and other characteristics that include the angle between its connected links. The possible node types for each degree are summarized in Table 1, and the detailed process can be found in [16].

The graph originally obtained may include some errors regarding the node types. For this reason, we propose some improvements that, in addition to avoiding theses errors, are able to reduce the number of unknown points compared to the previous approach [16]. The improvements are detailed as follows: 


\begin{tabular}{l|l}
\hline Degree & Possible types of nodes \\
\hline Nodes of degree 2 & $\begin{array}{l}\text { Connecting point } \\
\text { Meeting point }\end{array}$ \\
\hline Nodes of degree 3 & $\begin{array}{l}\text { Meeting point } \\
\text { Bifurcation point }\end{array}$ \\
\hline Nodes of degree 4 & $\begin{array}{l}\text { Meeting point } \\
\text { Bifurcation point } \\
\end{array}$ \\
Crossing point \\
\hline Nodes of degree 5 & Crossing point \\
\hline
\end{tabular}

Table 1 Possible types of nodes and corresponding degrees.

- Nodes of degree 2 and 3: The central retinal artery and vein emerge from the optic nerve, and bifurcate into several branches [35]. These branches of arteries and veins alternate along the retinal surface. Based on that, we introduced the concept of almost parallel vessels by including a condition to check if the angle between links is $\leq 15^{\circ}$ (see Figure $2(\mathrm{a})$ ). If this condition is met, the node is classified as a meeting point in order to distinguish between the artery link and the vein link.

- Nodes of degree 2: As stated before, the central artery and vein bifurcate into several branches over the retinal surface, but they never converge. Therefore, if two vessels converge at some point of the retinal surface, they correspond to different types. Based on that, we introduced the concept of link convergence by including a condition to the check if the angle between links is $\leq 90^{\circ}$ and is oriented to the optic disc (see Figure 2(b)). If this condition is met, the node is classified as meeting point in order to distinguish between the artery link and the vein link.

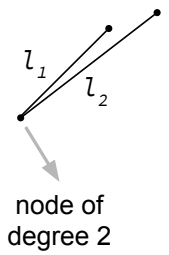

degree 2

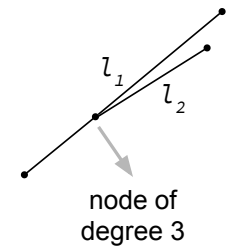

(a)

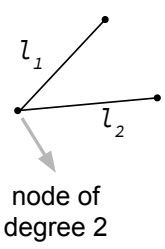

(b)

Fig. 2 (a) Nodes of degree 2 and 3 with almost parallel vessels, $l_{1}$ and $l_{2}\left(\right.$ angle $\left.\leq 15^{\circ}\right)$. (b) Node of degree 2 with an angle $\leq 90^{\circ}$ oriented to the optic disc.

\subsection{Multilevel thresholding}

After computing the local contrast and the graph, the average contrast of each graph link (segment) is computed as: 


$$
C_{s}=\frac{1}{N} \sum_{(i, j) \in s} C[i, j]
$$

where $N$ is the number of pixels $(i, j)$ that belongs to the segment $s$, and $C[i, j]$ refers to the local contrast (Eq. 1).

Given that the local contrast on the veins is higher than the local contrast on the arteries, we propose to use this feature to classify the graph segments into arteries and veins. For this purpose, we applied a multilevel thresholding based on the Otsu's method [36]. The idea is to consider the local contrast of the graph segments, and compute the histogram of each contrast level. Then, the Otsu's algorithm is applied to search the two thresholds $\left(t h_{1}\right.$ and $t h_{2}$ ) that minimize the intra-class contrast variance. As in this step we aim at achieving a robust classification, $t h_{1}$ and $t h_{2}$ should be sufficiently low and high, respectively, to get the minimum amount of false negatives.

Based on the thresholds $t h_{1}$ and $t h_{2}$, a partial classification $(P C)$ is computed with three target classes: artery, vein, and uncertain. In parallel, an average threshold is computed as $t h_{\mu}=\frac{t h_{1}+t h_{2}}{2}$, to avoid any unclassified segment and guarantee a full coverage rate at some point in the process. In this case, a total classification $(T C)$ is achieved by classifying all the segments into one of the two main classes: artery and vein. Figure 3 depicts this process.

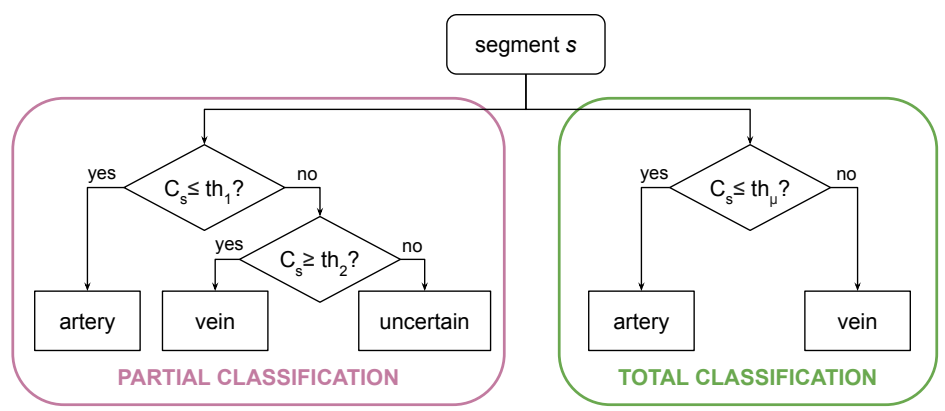

Fig. 3 Flowchart for segment classification: the partial classification is characterized by being robust, but at the expense of some unclassified segments (uncertain); whilst the total classification guarantees a full coverage rate.

As stated before, the background brightness varies along the retinal image due to light reflection changes from the spherical-shaped eye surface. This affects the local brightness and contrast of the vessel pixels, and can be easily confirmed by inspecting the image mean intensity on the four quadrants centered at the OD, or by observing the decreasing intensity when moving away from the OD. For these reasons, the multilevel thresholding is not applied to the graph segments of the whole image, but to the segments located at different regions of interest. In particular, we consider two different regions of interest (see Figure 4): four quadrants $Q$ centered at the OD, and three bands $B$ with different distances to the OD. Therefore, if we combine these two options for 
the regions of interest with the two approaches for segment classification, we obtain four A/V classified images: $P C Q$ and $P C B$ as partial classifications for quadrants and bands, respectively; and, equivalently, $T C Q$ and $T C B$ as total classifications.

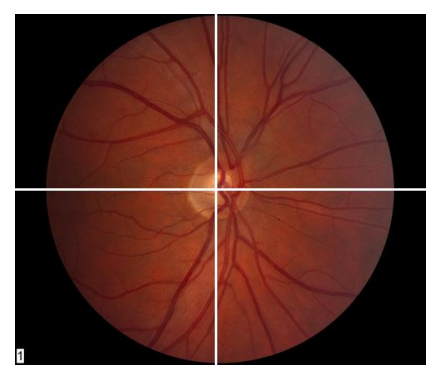

(a)

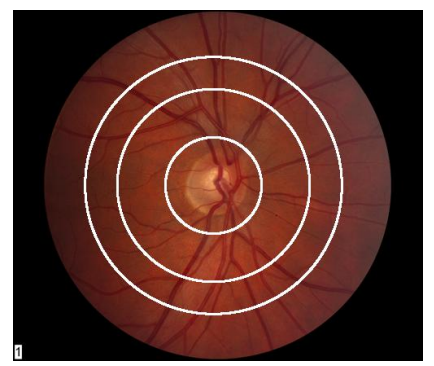

(b)

Fig. 4 Regions of interest: (a) four quadrants centered at the optic disc, (b) three bands with different distances to the optic disc.

With the main aim of benefiting from both approaches, and given the heterogeneity of images and datasets, the four A/V classified images are combined as detailed in Algorithm 1. In this manner, we obtain two final classifications: a partial classification $(P C F)$ that includes three categories (artery, vein, and uncertain), and a total classification $(T C F)$ that only includes the two main categories (artery and vein). The first one can be defined as an initial, robust $\mathrm{A} / \mathrm{V}$ classification that is further completed by means of graph propagation. Note that this classification is definitive; i.e., it cannot be modified in further stages, thus avoiding any change due to possible inaccuracies in the graph computation. Given that in the graph propagation step (Section 2.6) not all the uncertain segments of the $P C F$ can be finally classified, $T C F$ is here defined to be used in case of uncertainty, thus allowing to achieve a fully coverage rate in $\mathrm{A} / \mathrm{V}$ classification. Note that in this case, and according to some preliminary experiments, the $T C Q$ is used in case of disagreements between both total classifications $(T C Q$ and $T C B)$.

\subsection{Graph propagation}

A novel algorithm for graph propagation is proposed to obtain the final $\mathrm{A} / \mathrm{V}$ classification, which consists in combining the information obtained in the previous steps; i.e., the node types of the graph (see Section 2.4), and the classified segments (see Section 2.5).

Given an initial classification for the graph segments, the algorithm for graph propagation is detailed as follows:

1. For each uncertain segment, get the classes of its two nodes using the following rules defined for the different node types: 


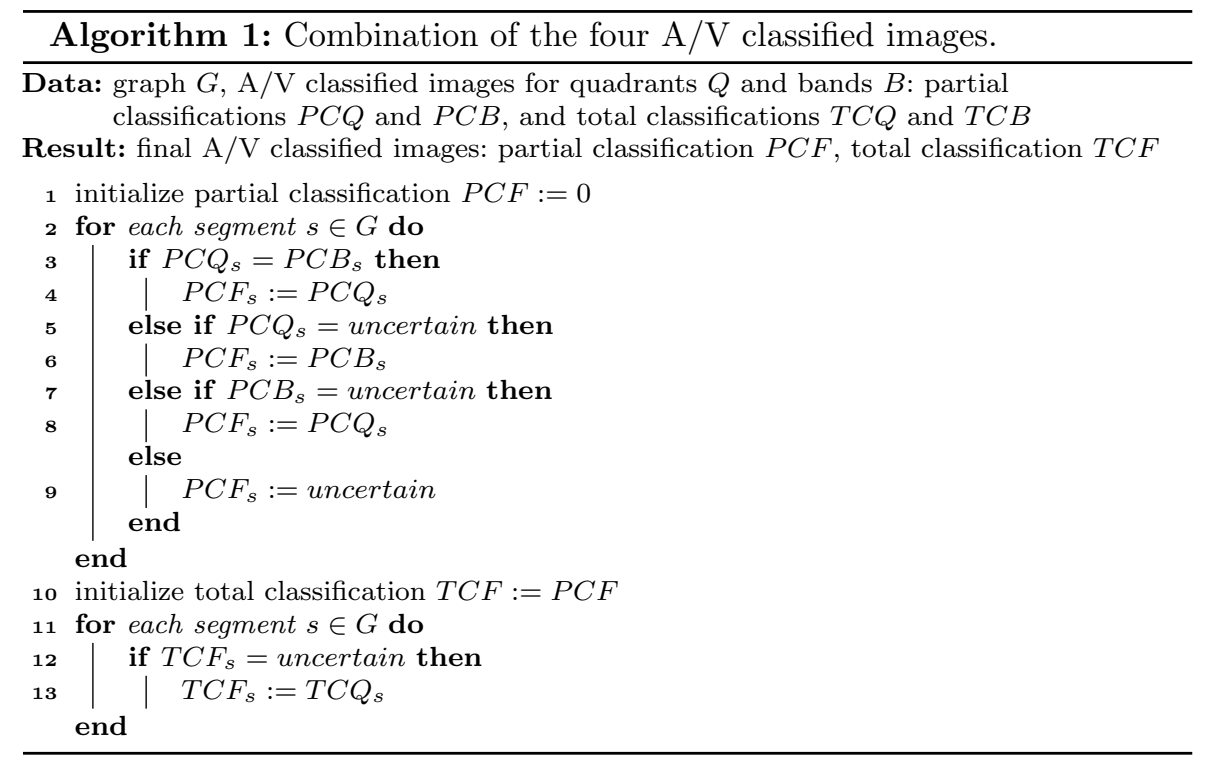

- Connecting point: There is only one connected link, so the class of the node is the class of the connected link.

- Bifurcation point: There are two connected links, so the class of the node depends on their classes: 1) if one link is labeled as uncertain, then the class of the node is the class of the other link; 2) if both links are labeled equally, then the class of the node is their class; 3) otherwise, the class of the node is uncertain.

- Meeting point with two adjacent links: There is only one connected link, so the class of the node is the opposite of the connected link.

- Meeting point with three adjacent links: Using angles and distances between the adjacent links, we determine which one is the meeting link. Based on that, we apply the rules for connecting and meeting points with two adjacent links, as appropriate.

- Crossing point: Using angles and distances between the adjacent links, we determine which one is the connecting link. Based on that, we apply the rules for connecting and bifurcation points, as appropriate.

2. For each uncertain segment, if both node classes are the same, then apply the propagation by labeling the segment with the corresponding class.

3. Repeat steps 1 and 2 until no more graph segments are re-labeled as artery or vein.

Next, the partial and total classifications obtained in Section 2.5 are used to apply the graph propagation algorithm as detailed in the following:

1. Use the partial classification (PCF) to initially label all the segments as artery, vein or uncertain.

2. Apply the algorithm for graph propagation. 
3. Use the total classification (TCF) to label the fully uncertain segments (i.e., those segments with the two nodes labeled as uncertain).

4. Apply the algorithm for graph propagation.

5. Apply the algorithm for graph propagation but also spreading to the partially uncertain segments (i.e., those segments with one of the two nodes labeled as uncertain), using the label of the known node.

6. Use the total classification (TFC) to label all the remaining uncertain segments.

Notice that by partially applying the process previously described, we can achieve different coverage rates in the A/V classification. Figure 5 illustrates this process, which can be divided in four different versions, from v.1 that corresponds to the minimum coverage rate to v.4 that guarantees a full coverage. These four versions will be referred in the analysis of the results.

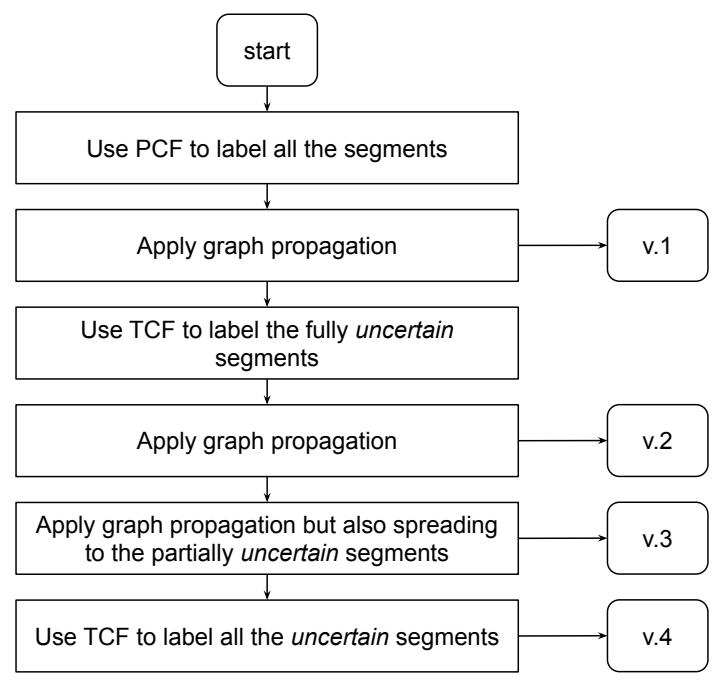

Fig. 5 Flowchart for graph propagation: the different levels of propagation together with the use of the partial and total classifications allow us to achieve different coverage rates in the final $\mathrm{A} / \mathrm{V}$ classification.

\subsection{ROI definition and AVR estimation}

AVR is computed from the vessel calibers inside a standard region of interest (ROI), a ring-shaped area within 0.5 to $1.0 \mathrm{OD}$ diameter from the OD boundaries [37].

The AVR index is defined as the quotient between two values, which are calculated using the Knudtson's revised formula [37]: the central retinal artery equivalent (CRAE) and the central retinal vein equivalent (CRVE). For AVR 
measurement, an approach similar to the one proposed by Niemeijer el al. [23] was applied: 1) six regions are considered to obtain distinct AVR regional values, based on the six largest arteries and veins of each region; and 2) the AVR index for the whole image is computed as the average of the six regional values.

\section{Results and discussion}

In this section, we present the results obtained by applying the proposed method to two different datasets, as described below. Additionally, we report some comparative studies with other approaches as well as qualitative results in terms of the graph network.

\subsection{INSPIRE dataset}

The INSPIRE dataset [30] is composed of 40 eye fundus images with a spatial resolution of $2392 \times 2048$ pixels, which are optic disc-centered. It includes two AVR measures for each image computed by two ophthalmologists using a semi-automated computer program developed at the University of Wisconsin. Additionally, a manual classification of the arteriolar-to-venular tree has been considered as the ground truth for the A/V classification. It was obtained by labeling each vessel pixel with one of the following labels: artery, vein, and uncertain.

In order to provide a comparative analysis with other $\mathrm{A} / \mathrm{V}$ classification methods applied to the INSPIRE dataset, we considered seven state-of-the-art approaches $[13,16,17,20,23,29,38]$. Table 2 depicts the confusion matrix that can be obtained as part of their experimental procedures.

\begin{tabular}{l|ccc}
\hline & \multicolumn{3}{|c}{ A/V reference } \\
A/V algorithm & Artery & Vein & Uncertain \\
\hline Artery & $T A$ & $F A$ & - \\
Vein & $F V$ & $T V$ & - \\
Uncertain & $U$ & $U$ & - \\
\hline
\end{tabular}

Table 2 Confusion matrix for the INSPIRE dataset.

The terms of the confusion matrix are used to define the performance metrics considered in the evaluation process, detailed as follows:

- Accuracy (Acc.): it represents the proportion of correctly classified pixels, among all the classified pixels.

$$
\text { Acc. }=\frac{T A+T V}{T A+F A+F V+T V}
$$

Note that both centerline accuracy (CA) and pixel-wise accuracy (PA) were calculated. 
- Sensitivity (Sens.): it represents the proportion of positives (veins) correctly classified.

$$
\text { Sens. }=\frac{T V}{T V+F V}
$$

- Specificity (Spec.): it represents the proportion of negatives (arteries) correctly classified.

$$
\text { Spec. }=\frac{T A}{T A+F A}
$$

- Vessel caliber $(v c)$ : it makes reference to the caliber of the vessel pixels considered in the evaluation. Therefore, $v c>0$ means that all the vessel pixels are taken into account.

Table 3 shows the results achieved with the proposed method and, as stated before, includes a comparison with seven state-of-the-art approaches, some of them supervised and others unsupervised. Analyzing the individual results in terms of the different vessel calibers, it can be observed that, in general, the pixel-wise accuracy (PA) is greater than the centerline accuracy (CA). This fact shows that the three automatic methods that provide the accuracy rates in this manner tend to classify correctly the largest vessels. Therefore, there is an increase in the accuracy rates when all the vessel pixels are considered instead of just those on the centerline. These two methods, Dashtbozorg [29] and Dashtbozorg et al. [16], are quite similar in terms of image features, with the main difference in terms of the learning process: the first one is an unsupervised method, whilst the second one is supervised. If these two approaches are compared, it can be observed that the supervised method slightly outperforms the unsupervised one for all vessel calibers, except the largest one ( $v c>20$ pixels). However, the unsupervised approach has the main benefit of be independent of a previous image labeling since no training phase is needed. If we include the proposed method in the comparisons, it outperforms the unsupervised method in most cases ( $v c>5,10,15$ and 20 pixels). Besides, it also outperforms the supervised approach when considering the three largest vessel calibers and provides accuracy rates over $96 \%$ in the best case. We note that these results are particularly relevant because a key requirement for AVR calculation is the correct classification of the main (largest) vessels. Furthermore, the proposed method has the same advantage of the unsupervised one: it does not require training, which means stability and independence of the image dataset. With respect to the other methods, they do not provide results in terms of vessel calibers, limiting the comparative analysis. Huang et al. [13] provides the highest accuracy rate in terms of CA, but at the expense of 100 features and a supervised classifier, thus limiting the generalization to new datasets. In terms of sensitivity and specificity, the most competitive results are achieved with the method proposed by Zhao et al. [20]. However, the authors do not analyze how their algorithm behaves with the largest vessels, since they do not estimate the AVR index.

Figure 6a,b illustrates the qualitative results of two sample images from the INSPIRE dataset. The results of the proposed A/V classification method 


\begin{tabular}{|c|c|c|c|c|c|c|c|}
\hline Method & $\mathrm{S} / \mathrm{U}$ & $\begin{array}{l}\text { Num. } \\
\text { feats. }\end{array}$ & $\begin{array}{l}\text { Vessel } \\
\text { caliber }\end{array}$ & $\begin{array}{l}\text { Acc. } \\
\text { CA } \\
\end{array}$ & $\mathrm{PA}$ & Sens. & Spec. \\
\hline \multirow[t]{5}{*}{ Proposed method (v.4) } & $\mathrm{U}$ & 1 & $v c>0 \mathrm{px}$ & 0.79 & 0.84 & 0.91 & 0.79 \\
\hline & & & $v c>5 \mathrm{px}$ & 0.84 & 0.88 & - & - \\
\hline & & & $v c>10 \mathrm{px}$ & 0.91 & 0.92 & - & - \\
\hline & & & $v c>15 \mathrm{px}$ & 0.93 & 0.94 & - & - \\
\hline & & & $v c>20 \mathrm{px}$ & 0.96 & 0.96 & - & - \\
\hline \multirow[t]{5}{*}{ Dashtbozorg [29] } & $\mathrm{U}$ & 30 & $v c>0 \mathrm{px}$ & 0.82 & 0.86 & 0.85 & 0.80 \\
\hline & & & $v c>5 \mathrm{px}$ & 0.83 & 0.86 & - & - \\
\hline & & & $v c>10 \mathrm{px}$ & 0.87 & 0.89 & - & - \\
\hline & & & $v c>15 \mathrm{px}$ & 0.90 & 0.91 & - & - \\
\hline & & & $v c>20 \mathrm{px}$ & 0.94 & 0.95 & - & - \\
\hline Estrada et al. $[17]$ & $\mathrm{U}$ & 3 & $v c>0 \mathrm{px}$ & 0.91 & - & 0.92 & 0.90 \\
\hline Zhao et al. [20] & $\mathrm{U}$ & 23 & $v c>0 \mathrm{px}$ & - & - & 0.96 & 0.97 \\
\hline Lyu et al. [38] & $\mathrm{U}$ & - & $v c>0 \mathrm{px}$ & 0.85 & - & 0.79 & 0.90 \\
\hline \multirow[t]{5}{*}{ Dashtbozorg et al. [16] } & $\mathrm{S}$ & 30 & $v c>0 \mathrm{px}$ & 0.85 & 0.88 & 0.91 & 0.86 \\
\hline & & & $v c>5 \mathrm{px}$ & 0.87 & 0.89 & - & - \\
\hline & & & $v c>10 \mathrm{px}$ & 0.90 & 0.91 & - & - \\
\hline & & & $v c>15 \mathrm{px}$ & 0.93 & 0.93 & - & - \\
\hline & & & $v c>20 \mathrm{px}$ & 0.93 & 0.93 & - & - \\
\hline Huang et al. [13] & $\mathrm{S}$ & 100 & $v c>0 \mathrm{px}$ & 0.92 & - & 0.90 & 0.91 \\
\hline Niemeijer et al. [23] & $\mathrm{S}$ & 27 & $v c>0 \mathrm{px}$ & - & - & 0.78 & 0.78 \\
\hline
\end{tabular}

Table 3 INSPIRE dataset: results of the proposed method compared with other stateof-the-art approaches for $\mathrm{A} / \mathrm{V}$ classification. S/U stands for supervised and unsupervised methods, respectively; and Num. feats. refers to the number of image features used for the classification.

can be visually compared with the manual labeling (ground truth images). As can be observed, the partial classification provides a robust, preliminary categorization of the blood vessels into arteries (blue), veins (red), and uncertain (green). These initial labels are next spread trough the graph by means of the different propagation stages, in order to achieve the final classification into arteries and veins.

Table 4 summarizes some AVR statistics for the complete INSPIRE dataset, including the two human observers and two previous approaches [23,25], in addition to our method. As can be seen, the mean errors generated by comparing the automatic results to the reference ones are very similar, regardless the approach considered. In order to statistically analyze them, the Friedman test has been applied [39]. It is a non-parametric test equivalent to ANOVA, but which does not require that some assumptions, such as normal distribution of the data, are met. Thus, the Friedman test was applied using two different error sets: the errors of the observer 2 and the three automatic methods compared to the observer 1 , and the errors of the observer 1 and the three automatic methods compared to the observer 2. According to the results obtained in both cases, there are no significant differences among the different automatic approaches and the two observers, thus demonstrating the adequacy of the proposed method for the automatic AVR calculation. 


\begin{tabular}{llllll}
\hline Method & Measure & Mean & St. deviation & Maximum & Minimum \\
\hline \hline Observer 1 & AVR & 0.67 & 0.08 & 0.93 & 0.52 \\
\hline Observer 2 & AVR & 0.66 & 0.08 & 0.85 & 0.45 \\
& E & 0.05 & 0.05 & 0.29 & 0.00 \\
\hline Proposed method (v.4) & AVR & 0.64 & 0.08 & 0.91 & 0.53 \\
& E1 & 0.06 & 0.07 & 0.28 & 0.00 \\
& E2 & 0.06 & 0.05 & 0.23 & 0.00 \\
\hline Niemeijer et al. $[23]$ & AVR & 0.67 & 0.07 & 0.81 & 0.55 \\
& E1 & 0.06 & 0.04 & 0.15 & 0.01 \\
& E2 & 0.06 & 0.06 & 0.28 & 0.00 \\
\hline Dashtbozorg et al. $[25]$ & AVR & 0.65 & 0.07 & 0.82 & 0.49 \\
& E1 & 0.05 & 0.04 & 0.16 & 0.00 \\
& E2 & 0.05 & 0.05 & 0.22 & 0.00 \\
\hline
\end{tabular}

Table 4 INSPIRE dataset: arteriolar-to-venular ratios (AVR) and errors (E), considering two human observers. Note that E1 and E2 refer to the errors with respect to the observers 1 and 2 , respectively.

\subsection{DRIVE dataset}

The DRIVE dataset [40] is composed of 40 retinal images, which are foveacentered and were captured with a resolution of $565 \times 584$ pixels. It includes manual segmented images for validation purposes and, based on them, the RITE dataset was created in [41] to provide a A/V reference standard, which has been considered in this research. Note that this standard was generated by labeling each vessel pixel with one of the following options: artery, vein, overlap, and uncertain.

As with the INSPIRE dataset, we carried out a comparative analysis with other A/V classification methods applied to the DRIVE dataset. In this case, we considered eight state-of-the-art approaches [13,16-18,20,23,29,38]. Table 5 depicts the confusion matrix that can be obtained as part of their experimental procedures.

\begin{tabular}{l|cccc}
\hline & \multicolumn{4}{|c}{ A/V reference } \\
A/V algorithm & Artery & Vein & Overlap & Uncertain \\
\hline Artery & $T A$ & $F A$ & $T A_{g}$ & - \\
Vein & $F V$ & $T V$ & $T V_{g}$ & - \\
Uncertain & $U$ & $U$ & $U$ & - \\
\hline
\end{tabular}

Table 5 Confusion matrix for DRIVE dataset.

The proposed method was evaluated using the following performance measures (see Section 3.1): centerline accuracy (CA), pixel-wise accuracy (PA), sensitivity (Sens.), and specificity (Spec). Two additional metrics suggested by $\mathrm{Hu}$ et al. [18] were also considered:

- Coverage rate: it represents the ratio of vessel pixels classified as arteries and veins $(\mathrm{A} / \mathrm{V}$ algorithm) over all the vessel pixels labeled in the ground 
truth, except the ones labeled as uncertain (A/V reference).

$$
\text { Coverage rate }=\frac{T A+F A+T A_{g}+F V+T V+T V_{g}}{T A+F A+T A_{g}+F V+T V+T V_{g}+U}
$$

- Overall accuracy $(\mathrm{OA})$ : it represents the pixel-wise accuracy but also including the terms $T A_{g}$ and $T V_{g}$.

$$
\mathrm{OA}=\frac{T A+T A_{g}+T V+T V_{g}}{T A+F A+T A_{g}+F V+T V+T V_{g}}
$$

Table 6 depicts the results obtained with the proposed method compared with eight state-of-the-art approaches. The results achieved with the proposed method demonstrate its robustness, with really similar performance values regardless the coverage rate, which ranges from $93.79 \%$ to $100.00 \%$ depending on the version applied (see Section 2.6). In addition, these results are also comparable with those obtained for the INSPIRE dataset, which show the adequacy of our method to the problem at hand, regardless of the dataset. In this sense, it is worth noting the poor performance of the approach proposed by Huang et al. [13] with this dataset, with an accuracy of 0.72 compared with the 0.92 achieve with INSPIRE. The rest of the methods included in the comparison present similar performances with a full coverage rate $(100 \%)$, but the approach proposed by $\mathrm{Hu}$ et al. [18]. Given that Hu's method does not provide a full coverage rate, it allows us to do a deeper analysis in terms of this metric. When using all the steps of our method, i.e. version v.4, no vessels remain unlabeled and so the coverage rate is $100 \%$, against the $83.55 \%$ of the Hu's method. In particular, the difference in terms of accuracy is of $3.71 \%$. A lower coverage rate can be achieved with the proposed method by considering the different alternatives or versions. Notice that each one implies less computation and so a reduction of the coverage rate. As expected, the lower the coverage rate, the greater the accuracy. More specifically, the method is able to provide a maximum accuracy of $83.53 \%$, slightly lower than the one obtained with the Hu's method, even though the coverage remains above the $93.5 \%$, i.e. over a $10 \%$ higher.

The nature of our method does not allow getting a coverage rate as low as the Hu's method, and so the results above are not directly comparable. In order to shed more light on the results obtained with DRIVE, we used three new metrics defined from the same confusion matrix depicted in Table 5:

- Global accuracy: the percentage of correctly classified vessel pixels among all the pixels detected in the segmentation process.

$$
\mathrm{G}-\mathrm{acc}=\frac{T A+T A_{g}+T V+T V_{g}}{T A+F A+T A_{g}+F V+T V+T V_{g}+U}
$$

- Global error: the complementary percentage of the accuracy. It can be divided into the percentage of wrongly classified vessel pixels and the percentage of unclassified ones, which are respectively: 


\begin{tabular}{|c|c|c|c|c|c|c|c|c|}
\hline Method & $\mathrm{S} / \mathrm{U}$ & $\begin{array}{l}\text { Num. } \\
\text { feats. }\end{array}$ & $\begin{array}{l}\text { CR } \\
(\%)\end{array}$ & $\begin{array}{l}\text { Acc. } \\
\text { CA }\end{array}$ & PA & $\mathrm{OA}$ & Sens. & Spec. \\
\hline \multirow{4}{*}{$\begin{array}{r}\text { Proposed method (v.1) } \\
\text { (v.2) } \\
(\mathrm{v} .3) \\
(\mathrm{v} .4)\end{array}$} & $\overline{\mathrm{U}}$ & 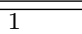 & 93.78 & 0.80 & 0.83 & 0.84 & 0.89 & 0.80 \\
\hline & $\mathrm{U}$ & 1 & 95.48 & 0.79 & 0.83 & 0.83 & 0.89 & 0.79 \\
\hline & $\mathrm{U}$ & 1 & 96.78 & 0.79 & 0.83 & 0.83 & 0.88 & 0.79 \\
\hline & $\mathrm{U}$ & 1 & 100 & 0.79 & 0.82 & 0.82 & 0.88 & 0.79 \\
\hline Estrada et al. [17] & $\mathrm{U}$ & 3 & 100 & 0.92 & - & - & 0.92 & 0.92 \\
\hline Zhao et al. [20] & $\mathrm{U}$ & 23 & 100 & - & - & - & 0.93 & 0.94 \\
\hline Dashtbozorg [29] & $\mathrm{U}$ & 30 & 100 & - & - & - & 0.87 & 0.84 \\
\hline Lyu et al. [38] & $\mathrm{U}$ & - & 100 & 0.83 & - & - & 0.87 & 0.78 \\
\hline Huang et al. [13] & $\mathrm{S}$ & 100 & 100 & 0.72 & $\overline{-}-$ & 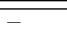 & 0.71 & 0.74 \\
\hline Dashtbozorg et al. [16] & $\mathrm{S}$ & 30 & 100 & 0.87 & - & - & 0.90 & 0.84 \\
\hline $\mathrm{Hu}$ et al. [18] & $\mathrm{S}$ & 31 & 83.55 & - & - & 0.86 & - & - \\
\hline Niemeijer et al. [23] & $\mathrm{S}$ & 27 & - & - & - & - & 0.80 & 0.80 \\
\hline
\end{tabular}

Table 6 DRIVE dataset: results of the proposed method compared with other state-of-theart approaches for $\mathrm{A} / \mathrm{V}$ classification. S/U stands for supervised and unsupervised methods, respectively; and Num. feats. refers to the number of images features used for the classification.

$$
\begin{aligned}
& \text { G-error }_{1}=\frac{F A+F V}{T A+F A+T A_{g}+F V+T V+T V_{g}+U} \\
& \text { G-error }_{2}=\frac{U}{T A+F A+T A_{g}+F V+T V+T V_{g}+U}
\end{aligned}
$$

Table 7 illustrates these new measures for the different approaches previously presented in Table 6 . As can be observed, the global accuracy of our method surpasses the previous approach [18] regardless the configuration. More specifically, the highest accuracy is obtained when applying all the steps of the proposed methodology (version v.4).

\begin{tabular}{lllll}
\hline Method & & G-acc (\%) & G-error 1 (\%) & G-error $2(\%)$ \\
\hline \hline Proposed method (v.1) & 78.33 & 15.45 & 6.22 \\
& (v.2) & 79.09 & 16.39 & 4.52 \\
& (v.3) & 80.05 & 16.73 & 3.22 \\
& (v.4) & 82.40 & 17.60 & 0.00 \\
\hline Hu et al. $[18]$ & & 71.94 & 11.61 & 16.45 \\
\hline
\end{tabular}

Table 7 DRIVE dataset: global accuracy and global error rates. The global error refers to the wrongly classified vessel pixels (G-error 1 ), and the unclassified ones (G-error 2 ).

Figure 6c,d depicts the qualitative results of two sample images from the DRIVE dataset. The ground truth images can be visually compared with the partial classifications, which include some uncertain segments (green), and the outcomes obtained after applying each propagation step, including the final classification that guarantees a full coverage rate.

\subsection{Graph computation results}

The graph propagation step is only applied to the segments initially labeled as uncertain. For this reason, the modifications proposed in the graph computa- 
tion step (see Section 2.4) only have a real impact in the final A/V classification when they correspond to uncertain segments. That is, we cannot measure the individual impact of the graph computation process on the final A/V classification results. For this reason, this section illustrates the improvements achieved with the graph modifications proposed in Section 2.4.

Figure 7 depicts five image samples from the two different datasets considered in the experimentation. Rows 1 and 2 show two images from the INSPIRE dataset, both of them with a node of degree 3 that has two almost parallel vessels (i.e., the angle between them is $\leq 15^{\circ}$ ). Based on this condition, the node is correctly classified as a meeting point, instead of a bifurcation point, thus allowing to distinguish between the artery and the vein. Rows 3 and 4 illustrate two images from the DRIVE dataset, both of them with a node of degree 2 that has two almost parallel vessels. As in the two previous samples, the node is correctly classified as a meeting point. Finally, row 5 depicts a node of degree 2 with a angle between segments $\leq 90^{\circ}$ ). In this case, the angle is oriented to the optic disc and so the node is correctly classified as a meeting point, allowing again to distinguish between the artery and the vein.

\section{Conclusions}

Given the relevance of A/V classification for the AVR estimation, this research presents a simple, yet powerful method for the automatic classification of retinal blood vessels. It makes a simplification of the classical approaches by using one single feature (the local contrast) and without any training step, thus facilitating its application to different retinal image datasets. Our approach also includes some improvements in the graph construction procedure, that make it more robust and reliable, and a novel graph propagation step that allows us to avoid errors in the final result caused by the graph when the initial $\mathrm{A} / \mathrm{V}$ classification was correct.

The proposed method has been validated on two different datasets. The INSPIRE dataset was first considered to analyze the accuracy of the vessel classification. Broadly speaking, the accuracy in main vessels (caliber $>10$ pixels) has been improved compared to both supervised and unsupervised approaches. Note the relevance of properly classifying main vessels since they are the ones used to compute the AVR. This dataset was also used to compute some AVR statistics, and the obtained results demonstrate that there are no significant differences between our approach and the human observers. The DRIVE dataset was also used for validation, and the experimentation carried out shows a higher coverage rate compared to other state-of-the-art approach, with notable improvements in terms of global accuracy.

Adding new image features to the proposed method requires a deep analysis focused on their individual and combined effect on both partial and final classifications. Our future research includes the study of other powerful features that can complete and/or complement the local contrast to improve the $\mathrm{A} / \mathrm{V}$ classification results. We also plan to consider different deep learn- 
ing techniques, including not only pixel-wise classification approaches but also semantic segmentation techniques.

\section{Acknowledgments}

Beatriz Remeseiro acknowledges the support of the Portuguese funding agency, FCT - Fundação para a Ciência e a Tecnologia, under Post-doctoral Fellowship program (ref. SFRH/BPD/111177/2015).

\section{References}

1. C.Y. Cheung, M.K. Ikram, R. Klein, T.Y. Wong. The clinical implications of recent studies on the structure and function of the retinal microvasculature in diabetes. Diabetologia 58(5), 871 (2015)

2. Y. Muraoka, A. Tsujikawa, K. Kumagai, M. Akiba, K. Ogino, T. Murakami, Y. AkagiKurashige, K. Miyamoto, N. Yoshimura. Age-and hypertension-dependent changes in retinal vessel diameter and wall thickness: an optical coherence tomography study. American Journal of Ophthalmology 156(4), 706 (2013)

3. R. Heitmar, G. Lip, R. Ryder, A. Blann. Retinal vessel diameters and reactivity in diabetes mellitus and/or cardiovascular disease. Cardiovascular Diabetology 16(1), 56 (2017)

4. J. Ding, K.L. Wai, K. McGeechan, et al. Retinal vascular caliber and the development of hypertension: a meta-analysis of individual participant data. Journal of Hypertension 32(2), 207 (2014)

5. V. Daien, I. Carriere, R. Kawasaki, J.P. Cristol, M. Villain, P. Fesler, K. Ritchie, C. Delcourt. Retinal vascular caliber is associated with cardiovascular biomarkers of oxidative stress and inflammation: the pola study. PloS One 8(7), e71089 (2013)

6. S.B. Seidelmann, B. Claggett, P. Bravo, A. Gupta, H. Farhad, M. Di Carli, S. Solomon. Retina vessel caliber in atherosclerotic cardiovascular event prediction: the atherosclerosis in communities study. Journal of the American College of Cardiology 67(13), 1893 (2016)

7. M.M. Fraz, P. Remagnino, A. Hoppe, B. Uyyanonvara, A.R. Rudnicka, C.G. Owen, S.A. Barman. Blood vessel segmentation methodologies in retinal images - A survey. Computer Methods and Programs in Biomedicine 108(1), 407 (2012)

8. A. Montoro, S. Morales, V. Naranjo, F. Lopez-Mir, M. Alcaniz. Feature extraction for retinal vascular network classification. in IEEE-EMBS International Conference on Biomedical and Health Informatics (2014), pp. 404-407

9. S. Irshad, M.U. Akram, S. Ayub, A. Ayaz. Retinal blood vessels differentiation for calculation of arterio-venous ratio. in International Conference Image Analysis and Recognition (2015), pp. 411-418

10. D. Relan, L. Ballerini, E. Trucco, T. MacGillivray, in Machine Intelligence and Signal Processing (Springer, 2016), pp. 77-84

11. X. Xu, W. Ding, M.D. Abràmoff, R. Cao. An improved arteriovenous classification method for the early diagnostics of various diseases in retinal image. Computer Methods and Programs in Biomedicine 141, 3 (2017)

12. S. Akbar, M.U. Akram, M. Sharif, A. Tariq, S.A. Khan. Decision support system for detection of hypertensive retinopathy using arteriovenous ratio. Artificial Intelligence in Medicine 90, 15 (2018)

13. F. Huang, B. Dashtbozorg, T. Tan, B.M. ter Haar Romeny. Retinal artery/vein classification using genetic-search feature selection. Computer Methods and Programs in Biomedicine 161, 197 (2018)

14. Q. Mirsharif, F. Tajeripour, H. Pourreza. Automated characterization of blood vessels as arteries and veins in retinal images. Computerized Medical Imaging and Graphics $\mathbf{3 7}(7), 607$ (2013) 
15. V.S. Joshi, J.M. Reinhardt, M.K. Garvin, M.D. Abramoff. Automated method for identification and artery-venous classification of vessel trees in retinal vessel networks. PloS One 9(2), e88061 (2014)

16. B. Dashtbozorg, A.M. Mendonça, A. Campilho. An automatic graph-based approach for artery/vein classification in retinal images. IEEE Transactions on Image Processing $\mathbf{2 3}(3), 1073$ (2014)

17. R. Estrada, M.J. Allingham, P.S. Mettu, S.W. Cousins, C. Tomasi, S. Farsiu. Retinal artery-vein classification via topology estimation. IEEE Transactions on Medical Imaging 34(12), 2518 (2015)

18. Q. Hu, M.D. Abràmoff, M.K. Garvin. Automated construction of arterial and venous trees in retinal images. Journal of Medical Imaging 2(4), 044001 (2015)

19. E. Pellegrini, G. Robertson, T. MacGillivray, J. van Hemert, G. Houston, E. Trucco. A graph cut approach to artery/vein classification in ultra-widefield scanning laser ophthalmoscopy. IEEE Transactions on Medical Imaging 37(2), 516 (2017)

20. Y. Zhao, J. Xie, H. Zhang, Y. Zheng, Y. Zhao, H. Qi, Y. Zhao, P. Su, J. Liu, Y. Liu. Retinal vascular network topology reconstruction and artery/vein classification via dominant set clustering. IEEE Transactions on Medical Imaging (2019)

21. M.I. Meyer, A. Galdran, P. Costa, A.M. Mendonça, A. Campilho. Deep convolutional artery/vein classification of retinal vessels. in International Conference Image Analysis and Recognition (2018), pp. 622-630

22. A. Galdran, M.I. Meyer, P. Costa, A.M. Mendonça, A. Campilho. Uncertainty-Aware Artery/Vein Classification on Retinal Images. in IEEE International Symposium on Biomedical Imaging (2019), pp. 556-560

23. M. Niemeijer, X. Xu, A.V. Dumitrescu, P. Gupta, B. van Ginneken, J.C. Folk, M.D. Abràmoff. Automated measurement of the arteriolar-to-venular width ratio in digital color fundus photographs. IEEE Transactions on Medical Imaging 30(11), 1941 (2011)

24. S. Vázquez, N. Barreira, M.G. Penedo, M. Rodríguez-Blanco. Reliable monitoring system for arteriovenous ratio computation. Computerized Medical Imaging and Graphics $\mathbf{3 7}(5), 337(2013)$

25. B. Dashtbozorg, A.M. Mendonça, A. Campilho. Assessment of Retinal Vascular Changes Through Arteriolar-to-Venular Ratio Calculation. in International Conference Image Analysis and Recognition (2015), pp. 335-343

26. W.A. Mustafa, H. Yazid, S.B. Yaacob. Illumination correction of retinal images using superimpose low pass and gaussian filtering. in International Conference on Biomedical Engineering (2015), pp. 1-4

27. E.S. Varnousfaderani, S. Yousefi, A. Belghith, M.H. Goldbaum. Luminosity and contrast normalization in color retinal images based on standard reference image. in Medical Imaging 2016: Image Processing, vol. 9784 (2016), vol. 9784, p. 97843N

28. F. Huang, B. Dashtbozorg, B.M. ter Haar Romeny. Artery/vein classification using reflection features in retina fundus images. Machine Vision and Applications 29(1), 23 (2018)

29. B. Dashtbozorg. Advanced Image Analysis for the Assessment of Retinal Vascular Changes, Ph.D thesis, Universidade do Porto. https://repositorioaberto.up.pt/handle/10216/78851?locale=en (2015)

30. M. Niemeijer, X. Xu, A. Dumitrescu, P. Gupta, B. van Ginneken, J. Folk, M. Abràmoff. INSPIRE-AVR: Iowa Normative Set for Processing Images of the Retina - Artery Vein Ratio. http://www.medicine.uiowa.edu/eye/inspire-datasets/ (2011)

31. B. Dashtbozorg, A.M. Mendonça, A. Campilho. Optic disc segmentation using the sliding band filter. Computers in Biology and Medicine 56, 1 (2015)

32. A.M. Mendonca, A. Campilho. Segmentation of retinal blood vessels by combining the detection of centerlines and morphological reconstruction. IEEE Transactions on Medical Imaging 25(9), 1200 (2006)

33. A.M. Mendonça, B. Remeseiro, B. Dashtbozorg, A. Campilho. Automatic and semiautomatic approaches for arteriolar-to-venular computation in retinal photographs. in SPIE Medical Imaging 2017: Computer-Aided Diagnosis, vol. 10134 (2017), vol. 10134, p. $101341 \mathrm{~L}$

34. M. Foracchia, E. Grisan, A. Ruggeri. Luminosity and contrast normalization in retinal images. Medical Image Analysis 9(3), 179 (2005) 
35. H. Schneiderman, The funduscopic examination (Butterworths, 1990)

36. N. Otsu. A threshold selection method from gray-level histograms. IEEE Transactions on Systems, Man, and Cybernetics 9(1), 62 (1979)

37. M.D. Knudtson, K.E. Lee, L.D. Hubbard, T.Y. Wong, R. Klein, B.E. Klein. Revised formulas for summarizing retinal vessel diameters. Current Eye Research 27(3), 143 (2003)

38. X. Lyu, Q. Yang, S. Xia, S. Zhang. Construction of retinal vascular trees via curvature orientation prior. in IEEE International Conference on Bioinformatics and Biomedicine (2016), pp. 375-382

39. J. Demšar. Statistical Comparisons of Classifiers over Multiple Data Sets. Journal of Machine Learning Research 7, 1 (2006)

40. M. Niemeijer, J.J. Staal, B.V. Ginneken, M. Loog, M.D. Abràmoff. DRIVE: Digital Retinal Images for Vessel Extraction. http://www.isi.uu.nl/Research/Databases/DRIVE (2004)

41. Q. Hu, M.K. Garvin, M.D. Abràmoff. RITE: Retinal Images vessel Tree Extraction. https://medicine.uiowa.edu/eye/rite-dataset (2015) 

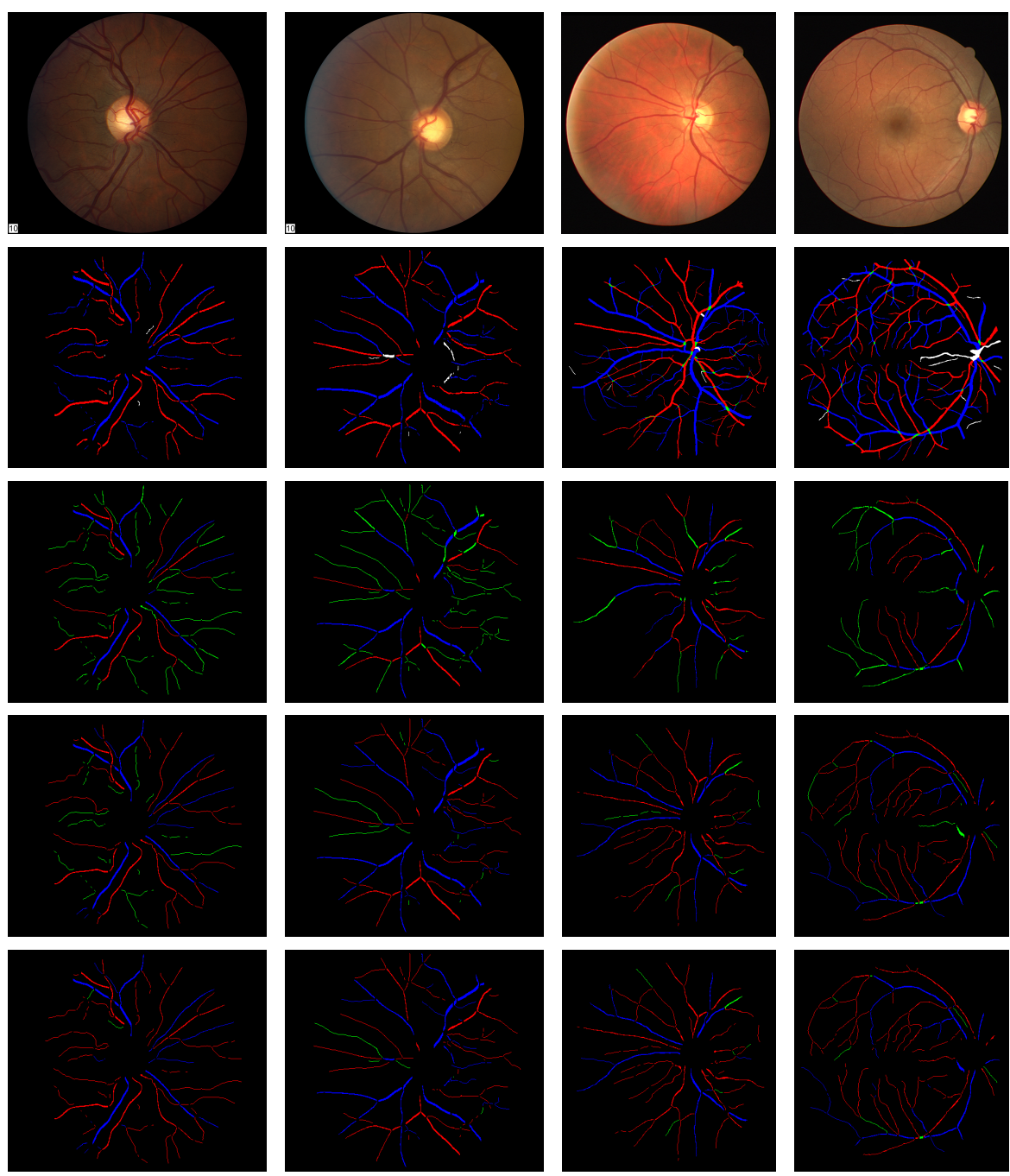

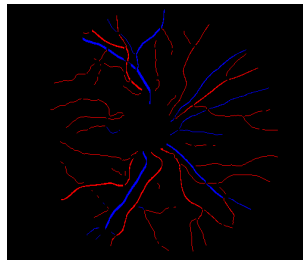

(a)

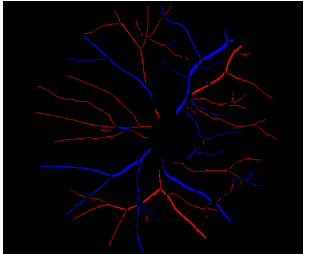

(b)

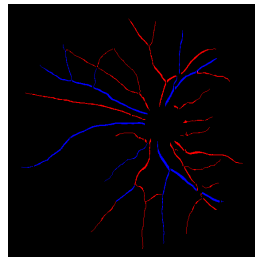

(c)

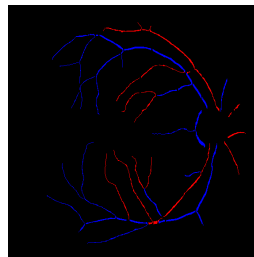

(d)

Fig. 6 (a,b) INSPIRE dataset, (c,d) DRIVE dataset. From top to bottom: original images, ground truth for $\mathrm{A} / \mathrm{V}$ classification, partial classification (PCF), initial stage of graph propagation (v.1), intermediate stages of graph propagation (v.2, v.3), and final classification after full graph propagation (v.4). Note that the intermediate stages (v.2, v.3) are shown together for the sake of simplicity. 

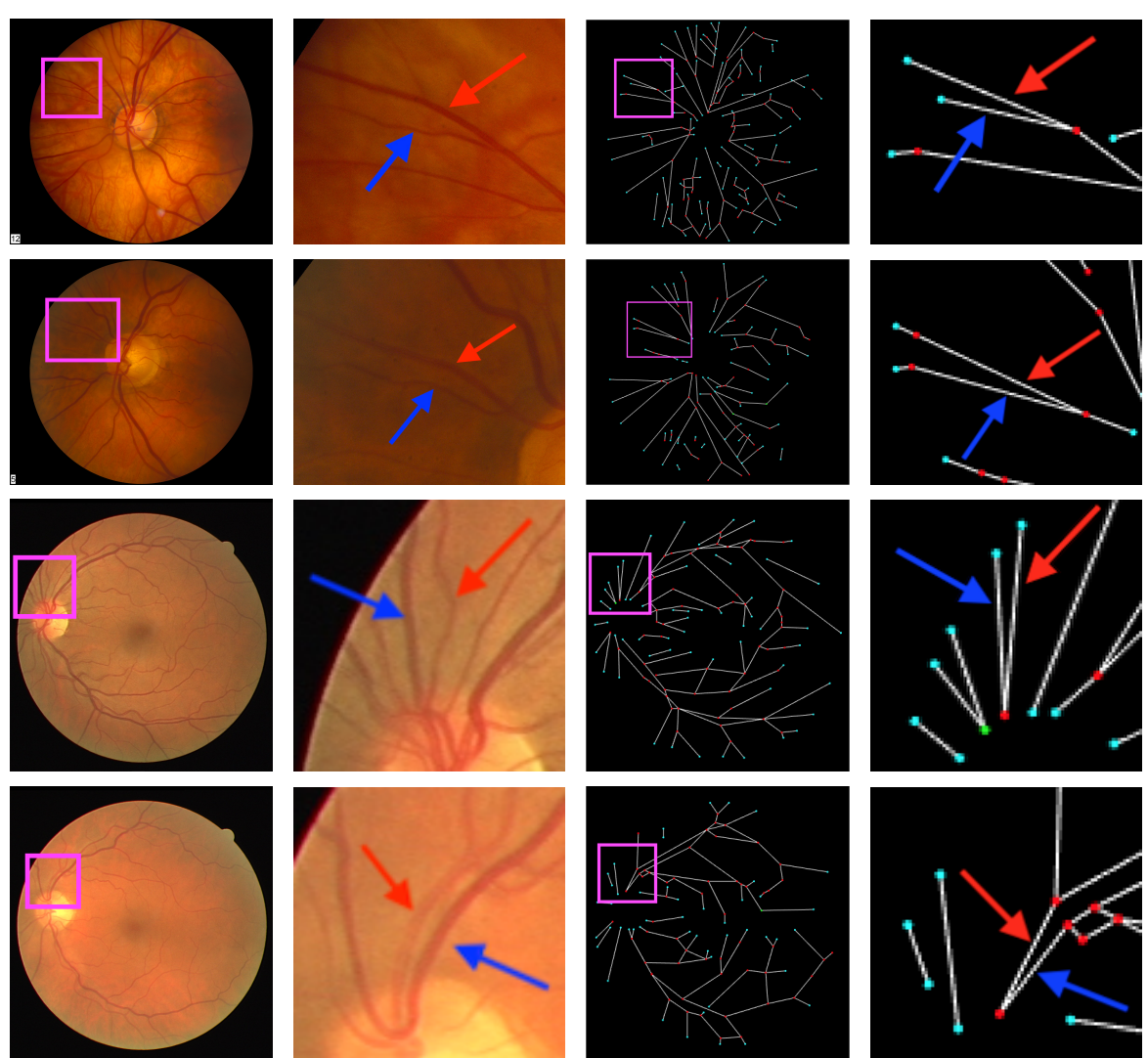

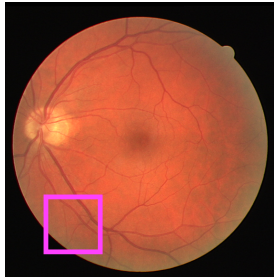

(a)

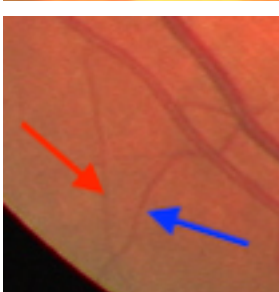

(b)

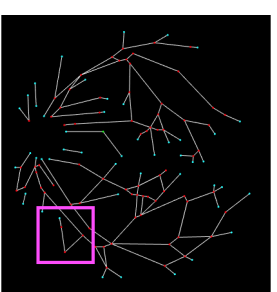

(c)

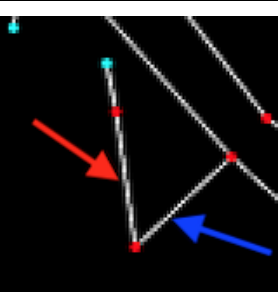

(d)

Fig. 7 Qualitative results of the graph computation step: (a) original image with the region of interest highlighted (magenta square), (b) zoomed region of interest of the original image, (c) computed graph with the region of interest highlighted (magenta square), and (d) zoomed region of interest of the graph. Blue arrows point to arteries, and red arrows to veins. The two top images are from the INSPIRE dataset, and the three bottom images are from the DRIVE dataset. 